\title{
Effects of Heparin on the Inhibitory Activities of Human Urinary Trypsin Inhibitor (Ulinastatin) on Trypsin, Chymotrypsin and Leukocyte Elastase
}

\author{
Tadahiro Shikimi, Keisuke Hattori and Shuji Takaori \\ Department of Pharmacology, Shimane Medical University, Enya, Izumo, Shimane 693, Japan \\ Received January 8, 1993 Accepted March 19, 1993
}

\begin{abstract}
Effects of heparin on the inhibitory activities of human urinary trypsin inhibitor (ulinastatin) on trypsin, chymotrypsin and leukocyte elastase were studied. Heparin per se neither influenced the enzymatic activities nor changed the mode of inhibition of ulinastatin on the enzymes. In the presence of heparin, inhibitory effects of ulinastatin on trypsin were enhanced, whereas its effects on chymotrypsin and elastase were attenuated. These results suggest that the two functional domains in ulinastatin are differently affected by heparin.
\end{abstract}

Keywords: Urinary trypsin inhibitor (human), Ulinastatin, Heparin

Ulinastatin (UT) and aprotinin, derived respectively from human urine and bovine lung, are used as remedies for acute pancreatitis. They display inhibitory effects on trypsin, chymotrypsin and leukocyte elastase $(1,2)$. Although both these inhibitors are highly homologous in their amino acid sequences (3), their physicochemical properties are strikingly different. Aprotinin is a basic polypeptide with a molecular weight of about $6.5 \mathrm{kDa}$ and shows an isoelectric point close to $\mathrm{pH} 10.5$ (2), whereas UT, besides displaying an isoelectric point of 2.6 (4), is an acidic mucoprotein with a molecular weight of $40-50 \mathrm{kDa}$ in SDS-polyacrylamide gel electrophoresis (5). UT has two functional domains: domain 1 ( $\mathrm{N}$-terminal domain) inhibits chymotrypsin and leukocyte elastase activities, and domain 2 (C-terminal domain) suppresses trypsin activity (3). Previously, we have found that heparin enhances the inhibitory effect of aprotinin on the esterolytic activity of trypsin without altering the effect on the esterolytic activity of chymotrypsin (6). This report showed that heparin differently influenced the UT activities on trypsin, chymotrypsin and leukocyte elastase.

The inhibitory activities of UT (a kind gift from Mochida Pharmaceutical Co., Tokyo) on trypsin (EC 3.4.21.4; 3 times recrystallized product from bovine pancreas, Miles Laboratories, Elkhart, IN, U.S.A.), chymotrypsin (EC 3.4.21.1; 3 times recrystallized product from bovine pancreas, Miles Laboratories) and human leukocyte elastase (EC 3.4.21.37; Sigma Chemical Co., St. Louis, MO, U.S.A.) were measured with synthetic sub- strates that were methylcoumarylamide derivatives. The reaction was carried out as follows: the reaction mixture (containing $50 \mu \mathrm{l}$ of $0.5 \mathrm{M}$ Tris- $\mathrm{HCl}$ buffer (pH 8), $50 \mu 1$ of $0.1 \mathrm{M} \mathrm{CaCl}_{2}, 100 \mu \mathrm{l}$ of distilled water, $100 \mu \mathrm{l}$ of UT solution and $100 \mu$ lof polysaccharide solution) was incubated with $50 \mu \mathrm{l}$ of the enzyme preparation at $37^{\circ} \mathrm{C}$ for $5 \mathrm{~min}$. Thereafter, $50 \mu$ of substrate solution was added to the incubation mixture, which was then allowed to incubate further with trypsin, chymotrypsin and elastase at $37^{\circ} \mathrm{C}$ for 30,5 and $60 \mathrm{~min}$, respectively. The enzymatically released 7-amino-4-methyl-coumarin (AMC) linearly increased during these reaction intervals. In the kinetic study, the reaction intervals used for trypsin, chymotrypsin and elastase were 15,5 and $15 \mathrm{~min}$, respectively. As the substrates for trypsin, chymotrypsin and elastase, $t$-butyloxycarbonyl-L-glutaminyl-L-alanyl-L-arginine 4-methylcoumaryl-7-amide (Boc-Gln-Ala-Arg-MCA), succinyl-Lalanyl-L-alanyl-L-prolyl-L-phenylalanine 4-methyl-coumaryl-7-amide (Suc-Ala-Ala-Pro-Phe-MCA) and $N$-methoxy-succinyl-L-alanyl-L-alanyl-L-prolyl-L-valine 4-methylcoumaryl-7-amide (Suc (OMe)-Ala-Ala-Pro-Val-MCA) were used, respectively. These synthetic substrates were products of Peptide Institute, Inc., Osaka. The reaction was stopped by the addition of $500 \mu 1$ of $20 \%$ acetic acid. Fluorescence intensities of the enzymatically released AMC in the reactions with trypsin, chymotrypsin and elastase were measured using excitation/emission wavelengths of $380 / 440 \mathrm{~nm}, 380 / 460 \mathrm{~nm}$ and $370 / 460$ $\mathrm{nm}$, respectively. As the polysaccharide, mucopolysaccha- 
rides such as heparin (sodium salt from porcine intestinal mucosa, 150 USP units per mg, Sigma Chemical Co.), heparan sulfate (sodium salt from bovine kidney, Seikagaku Kogyo Co., Tokyo), chondroitin sulfate B (sodium salt from porcine skin, Seikagaku Kogyo Co.), chondroitin sulfate $\mathrm{C}$ (sodium salt from shark cartilage, Seikagaku Kogyo Co.) and hyaluronic acid (sodium salt from porcine skin, Seikagaku Kogyo Co.) were used. Sodium dextran sulfate 5000 (Wako Pure Chemical Industries, Osaka) was also used as a sulfated polysaccharide.
Among the polysaccharides, heparin enhanced the inhibitory effect of UT on trypsin (Fig. 1a). However, the inhibitory effects of UT on chymotrypsin and elastase were attenuated in the presence of heparin (Fig. 1, b and c). Attenuation effects on UT activities were also observed with sulfated polysaccharides such as heparan sulfate, chondroitin sulfate $\mathrm{B}$, chondroitin sulfate $\mathrm{C}$ and dextran sulfate, accordingly (Fig. 1, b and c). However, hyaluronic acid, which lacks the sulfate groups, did not affect the UT activity. These results indicate that the functional

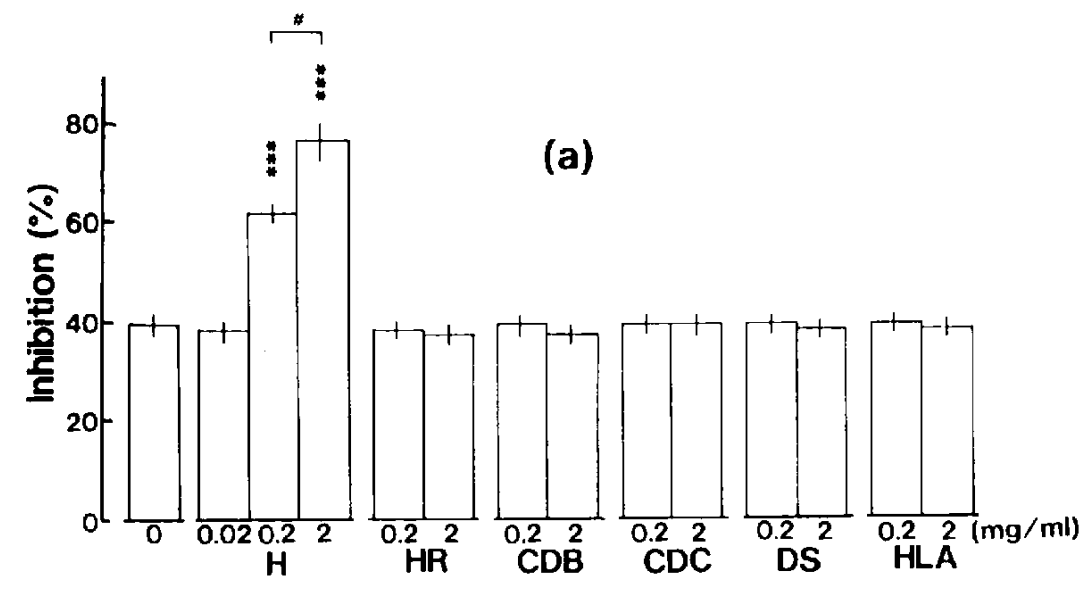

(b)

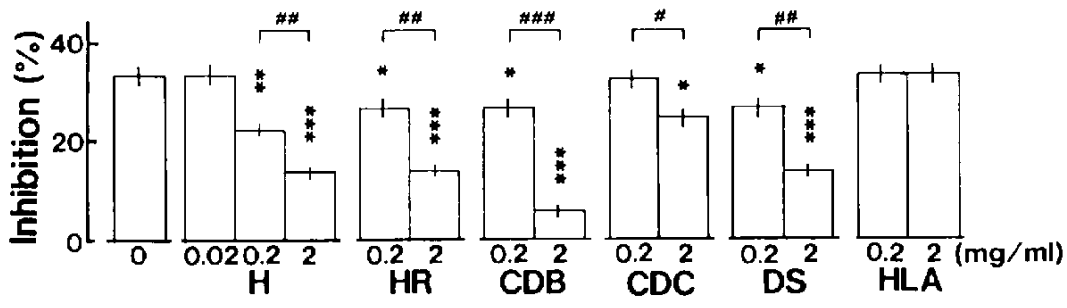

(c)

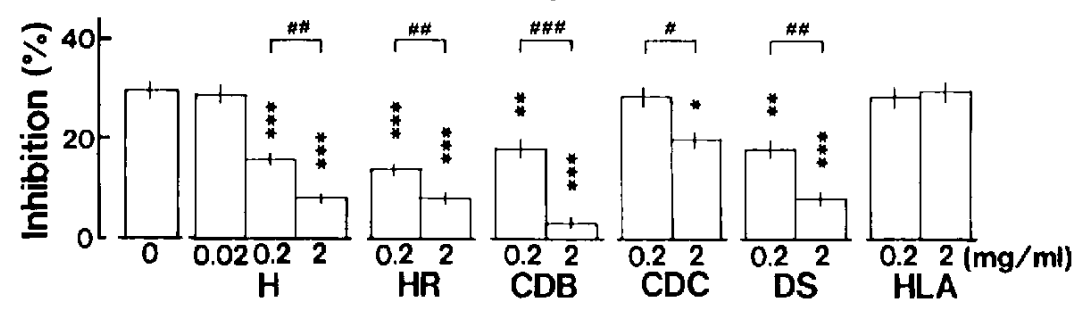

Fig. 1. Effects of polysaccharides on the inhibitory activities of ulinastatin (UT) on trypsin (a), chymotrypsin (b) and leukocyte elastase $(c)$. Trypsin $(6.25 \mathrm{ng} / \mathrm{ml})$, chymotrypsin $(100 \mathrm{ng} / \mathrm{ml})$ and leukocyte elastase $(0.4 \mu \mathrm{g} / \mathrm{ml})$ were incubated with various quantities of polysaccharides without and with UT of $24 \mathrm{ng} / \mathrm{ml}, 80 \mathrm{ng} / \mathrm{ml}$ and $2 \mu \mathrm{g} / \mathrm{ml}$ in (a), (b) and (c), respectively. Substrates for (a), (b) and (c) were Boc-Gln-Ala-Arg-MCA $\left(10^{-5} \mathrm{M}\right)$, Suc-Ala-Ala-Pro-Phe-MCA $\left(10^{-5} \mathrm{M}\right)$ and Suc (OMe)-AlaAla-Pro-Val-MCA $\left(2 \times 10^{-5} \mathrm{M}\right)$, respectively. The enzymatic activity without UT was taken as $100 \%$, and the inhibition $\%$ shown on the ordinate was calculated from the enzymatic activity with UT. Each column shows the mean value \pm S.E.M. for 4 experiments. Significant differences from the control value (polysaccharide concentration, $0 \mathrm{mg} / \mathrm{ml}$ ) (represented by the symbol $^{*}$ ) or from the value of corresponding column (represented by the symbol $\left.{ }^{*}\right)$ were determined by Student's $t$-test: $P<0.05\left({ }^{*},{ }^{*}\right)$, $\mathrm{P}<0.01\left(^{* *},{ }^{*}\right), \mathrm{P}<0.001\left(^{* * *}, *^{* * *}\right) . \mathrm{H}, \mathrm{HR}, \mathrm{CDB}, \mathrm{CDC}, \mathrm{DS}$ and HLA represent heparin, heparan sulfate, chondroitin sulfate $B$, chondroitin sulfate $C$, dextran sulfate and hyaluronic acid, respectively. 
Table 1. Kinetic coefficients of the interaction of trypsin, chymotrypsin and leukocyte elastase with ulinastatin in the presence or absence of heparin

\begin{tabular}{lccccc}
\hline Enzyme & Mode of inhibition & Heparin & $\mathrm{K}_{\mathrm{m}}(\mathrm{M})$ & $\mathrm{K}_{\mathrm{app}}(\mathrm{M})$ & $\mathrm{K}_{\mathrm{i}}(\mathrm{M})$ \\
\hline Trypsin & noncompetitive & $(-)$ & $2.0 \times 10^{-5}$ & $2.0 \times 10^{-5}$ & $7.8 \times 10^{-10}$ \\
& & $(+)$ & $2.0 \times 10^{-5}$ & $2.0 \times 10^{-5}$ & $3.7 \times 10^{-10}$ \\
Chymotrypsin & noncompetitive & $(-)$ & $10.0 \times 10^{-5}$ & $10.0 \times 10^{-5}$ & $3.7 \times 10^{-9}$ \\
& & $(+)$ & $10.0 \times 10^{-5}$ & $10.0 \times 10^{-5}$ & $7.3 \times 10^{-9}$ \\
Elastase & uncompetitive & $(-)$ & $9.1 \times 10^{-5}$ & $3.0 \times 10^{-5}$ & $2.5 \times 10^{-8}$ \\
& & $(+)$ & $9.1 \times 10^{-5}$ & $4.5 \times 10^{-5}$ & $4.9 \times 10^{-8}$ \\
\hline
\end{tabular}

The amounts of enzyme and ulinastatin (UT) and the substrate for the respective enzyme are similar to those indicated in Fig. 1. Each enzyme was preincubated at $37^{\circ} \mathrm{C}$ for $5 \mathrm{~min}$ with or without UT in the presence $(+)$ or absence $(-)$ of heparin $(0.2 \mathrm{mg} / \mathrm{ml})$, and various amounts of the respective substrate were then added. The enzymatically released AMC was measured with fluorophotometer. Kinetic coefficients were obtained from Lineweaver-Burk and Hofstee plots based on the data. $K_{\mathrm{m}}$ : Michaelis constant in the absence of UT. $K_{\mathrm{app}}$ : Apparent Michaelis constant in the presence of UT. $K_{i}$ : Inhibitor constant obtained in the noncompetitive inhibition for trypsin or chymotrypsin, and in the uncompetitive inhibition for elastase.

domains of UT, in which domain 1 (N-terminal domain) and 2 (C-terminal domain) inhibit chymotrypsin/leukocyte elastase and trypsin activities (3), respectively, are differently affected by heparin. With regards to the attenuation effects on the UT activity, chondroitin sulfate $C$ was less potent than chondroitin sulfate $B$ (compare CDC with $\mathrm{CDB}$ in Fig. 1, b and c). Thus, not only the sulfate groups but differences in uronic acid and the position of sulfate groups also influenced the attenuation effect.

As for the mode of inhibition of UT on trypsin, chymotrypsin and leukocyte elastase, inhibitions on trypsin and chymotrypsin were found to be noncompetitive, whereas that on elastase was uncompetitive (data not shown). This implicates that UT binds to trypsin (or chymotrypsin) and the trypsin - substrate (or chymotrypsin.substrate) complex, whereas UT per se does not bind to elastase, and instead it binds to the elastase-substrate complex. The inhibition mode of UT was not affected by heparin. From kinetic parameters of Lineweaver-Burk plots and Hofstee plots of the data (Table 1), heparin alone did not have any effects on the activities of trypsin, chymotrypsin and elastase (compare $\mathrm{K}_{\mathrm{m}}$ value with and without heparin in Table 1). Even in the presence of UT, heparin did not have any effects on the respective apparent $K_{m}$ value of trypsin and chymotrypsin (Table 1). The inhibitor constant $\left(K_{i}\right)$ values of UT for trypsin, chymotrypsin and elastase obtained in the absence of heparin were similar to those reported by Selloum et al. (7). In the presence of heparin, the $\mathrm{K}_{\mathrm{i}}$ value of UT for trypsin was decreased, whereas those for chymotrypsin and elastase were increased in the presence of heparin (Table 1). Thus, heparin enhanced the complex formation of trypsin.UT and trypsin-substrate-UT, whereas it de- creased the formation of complexes of chymotrypsin - UT and chymotrypsin-substrate.UT and that of elasatse. substrate.UT. The similar mechanism was observed regarding the enhancing effect of heparin on the inhibitory activity of aprotinin on trypsin (8).

Under the present experimental conditions (incubation of enzyme with UT and polysaccharide before addition of substrate and short reaction time), the quantity of heparin required for the enhancement or attenuation effect of UT activities was more than 100-fold higher than that of UT (see legend of Fig. 1). This might be attributed to the properties of UT and heparin: Both substances have an anionic character. UT is an acidic mucoprotein (3), and the carbohydrate content of UT, which is partially responsible for the anionic character of UT (7), is $35 \%$ (9) to $50 \%(10)$ of its molecular weight. Heparin is also an anionic polysaccharide due to its sulfate and carboxylic groups. However, our present results imply that the presence of heparin and sulfated mucopolysaccharides (in connective tissue and vascular endothelium) may have a modulatory effect on the protease inhibitory activity of UT systemically infused into the body for the treatment of acute pancreatitis and shock, in which the release of pancreatic enzymes can induce pancreatic damage and accumulation of plasma trypsin $(11,12)$. In addition, our results indicate that heparin may serve as a biochemical tool to modify or analyze the domain of UT which influences its biological functions, since the two functional domains of UT are differently affected by heparin.

\section{Acknowledgment}

This work was supported in part by Mita Foundation in Matsue, Japan. 


\section{REFERENCES}

1 Balduyck, M., Davril, M., Mizon, C., Smyrlaki, M., Hayem, A. and Mizon, J.: Human urinary proteinase inhibitor: Inhibitory properties and interaction with bovine trypsin. Biol. Chem. Hoppe Seyler 366, 9-14 (1985)

2 Fritz, H, and Wunderer, G.: Biochemistry and applications of aprotinin, the kallikrein inhibitor from bovine organs. Arzneimittleforschung 33, 479-494 (1983)

3 Gebhard, W. and Hochstrasser, K.: Inter- $\alpha$-trypsin inhibitor and its close relatives. In Proteinase Inhibitors, Edited by Barrett, A.J. and Salvesen, G., pp. 389-401, Elsevier Science Publishing Co., Amsterdam (1986)

4 Barthelemy-Clavey, V., Yapo, E.A., Vanhoutte, G., Hayem, A. and Mizon, J.: Purification et caracterisation des inhibiteurs de proteases de l'urine humaine. Biochim. Biophys. Acta 580, 154- 165 (1979) (Abs. in English)

5 Balduyck, M., Hayem, A., Kerckaert, J.P., Mizon, C. and Mizon, J.: Isolation of a human urinary trypsin inhibitor. Biochem. Biophys. Res. Commun. 109, 1247-1255 (1982)

6 Shikimi, T.: Enhancing effect of heparin on aprotinin activity. Experientia 37, 1179-1180 (1981)

7 Selloum, L., Davril, M., Mizon, C., Balduyck, M. and Mizon,
J.: The effect of the glycosaminoglycan chain removal on some properties of the human urinary trypsin inhibitor. Biol. Chem. Hoppe Seyler 368, 47-55 (1987)

8 Shikimi, T.: Immunochemical and kinetic evidence that heparin enhances aprotinin activity. Japan. J. Pharmacol. 38, 433-435 (1985)

9 Balduyck, K., Mizon, C., Loutf, M., Richet, C., Roussel, P. and Mizon, J.: The major human urinary trypsin inhibitor is a proteoglycan. Eur. J. Biochem. 158, 417-422 (1986)

10 Hochstrasser, K., Schönberger, Ö.L., Rossmanith, I. and Wachter, E.: Kunitz-type proteinase inhibitors derived by limited proteolysis of the inter- $\alpha$-trypsin inhibitor, V. Attachments of carbohydrates in the human urinary trypsin inhibitor isolated by affinity chromatography. Hoppe Seyers Z. Physiol. Chem. 362, 1357-1362 (1981)

11 Ohnishi, H., Kosuzume, H., Ashida, Y., Kato, K. and Honjo, I.: Effects of urinary trypsin inhibitor on pancreatic enzymes and experimental acute pancreatitis. Dig. Dis. Sci. 29, 26-32 (1984)

12 Ohnishi, H., Suzuki, K., Niho, T., Ito, C. and Yamaguchi, K.: Protective effects of urinary trypsin inhibitor in experimental shock. Japan. J. Pharmacol. 39, 137-144 (1985) 